\title{
Understanding and Managing Severe Asthma in the Context of COVID-19
}

\author{
Bárbara Kong-Cardoso (1D \\ Amélia Ribeiro ${ }^{2}$ \\ Rita Aguiar' \\ Helena Pité (iD) \\ Mário Morais-Almeida $\mathbb{D}^{\prime}$ \\ 'Allergy Center, CUF Descobertas \\ Hospital and CUF Tejo Hospital, Lisbon, \\ Portugal; ${ }^{2}$ Pneumology Department, \\ Centro Hospitalar Barreiro Montijo, \\ Barreiro, Portugal
}

\begin{abstract}
Coronavirus disease 2019 (COVID-19) continues to spread across the world. Since the beginning of the pandemic, the question of whether asthma is a risk factor for getting the infection or for poor outcomes motivated a great debate. In the field of severe asthma and its treatment during COVID-19 pandemic, several issues are also pending. A literature review focused on the management of severe asthma patients in the context of COVID-19 is performed. The available evidence suggests that severe asthma patients do not have an increased risk of poor COVID-19 outcomes and that it is safe to treat asthmatic patients with inhaled corticosteroids (ICS) and biologics during the pandemic, even though some studies indicate that high doses of ICS may predispose to COVID-19. The chronic use of oral corticosteroid (OCS) might be associated with poor COVID-19 outcomes, although there is no complete agreement. There is very limited evidence concerning the use of triple therapy for asthma in the context of this pandemic. Ultimately, severe asthma patients should maintain their medication during the COVID-19 pandemic, including biologic agents. More studies are needed to address the role of asthma medications and asthma's different phenotypes on the incidence and course of COVID-19.
\end{abstract}

Keywords: allergy, biologics, corticosteroids, COVID-19, SARS-CoV-2, severe asthma

\section{Introduction}

Coronavirus disease 2019 (COVID-19), caused by the Severe Acute Respiratory Syndrome CoronaVirus 2 (SARS-CoV-2), continues to spread across the world. As with other respiratory viruses, there were great concerns about the impact of COVID-19 in chronic respiratory diseases, particularly in asthmatics who might be at higher risk of infection and/or poor outcomes

Contrary to some expectations, in most studies, asthma was not associated with an increased risk of severe COVID-19. ${ }^{1}$ In the first studies, unlike old age and underlying morbidities such as cardiovascular diseases, in particular hypertension and metabolic disorders such as obesity and diabetes, asthma was not identified as a significant risk factor for COVID-19 morbidity or mortality. ${ }^{2-4}$ Studies from many countries on multiple continents (France, China, Italy, Sweden, Spain, Belgium, Israel, Mexico, Brazil, Saudi Arabia, and India) reported a lower prevalence of asthma among patients hospitalized with COVID-19 than in the general population in each region. ${ }^{1,5}$

Consequently, the Centers for Disease Control and Prevention (CDC), which initially considered patients with asthma to be at high risk for severe illness and mortality from COVID-19, revised their recommendations and now only consider those with moderate or severe asthma to be at high risk. ${ }^{6}$
Correspondence: Bárbara Kong-Cardoso CUF Descobertas Hospital and CUF Tejo Hospital, Lisbon, Portugal

Email barbarakc@gmail.com 
Studies from the USA, the UK, Ireland, Korea, and Australia showed a higher prevalence of asthma among patients hospitalized with COVID-19 and suggested that severe asthma patients were at higher risk of in-hospital mortality from COVID-19. ${ }^{1,5,7,8}$

Thus, the question of whether asthma is a risk factor for contracting the infection or associated with severe disease or negative outcomes motivated a great debate. Recent publications on this matter revealed a variety of results, gaining even more complexity with increasing data indicating that different phenotypes of asthma may be associated with different COVID-19 outcomes. ${ }^{1,9-12}$

The asthma treatments themselves are also a topic of debate, namely immunomodulating drugs, such as corticosteroids and biologic therapies, which one might expect to be related to an increased risk of infection or poor outcomes. ${ }^{13-15} \mathrm{Up}$ to $10 \%$ of the asthmatics have severe asthma and a clear understanding of the risk/benefit of maintaining treatment with immunomodulating drugs is essential to support correct treatment of these patients, who are most at risk of clinical decompensation. ${ }^{16}$ Currently approved biologics for the treatment of severe asthma are omalizumab (a recombinant DNA-derived humanized monoclonal antibody that selectively binds to human immunoglobulin E [IgE]), mepolizumab (a monoclonal antibody directed against interleukin-5 [IL-5]), benralizumab (a humanized fucosylated monoclonal antibody against IL5 alfa receptor [IL5Ra]), reslizumab (a monoclonal antibody against IL-5), and dupilumab (a humanized monoclonal antibody directed against the alpha-chain of the interleukin 4 receptor [IL4Ra]). Biologic therapy raised some concerns, particularly anti-IL5 and anti-IL5 receptor blocking monoclonal antibodies that induce an eosinophil depletion, considering that eosinopenia is a biomarker of COVID-19 severity. ${ }^{17}$

Conversely, it has been suggested that biologics may be effective against COVID-19 related asthma exacerbations, by reducing baseline airway inflammation and possibly through specific antiviral properties. ${ }^{18}$ In the PROSE (Preventative Omalizumab or Step-up Therapy for Severe Fall Exacerbations) study, omalizumab decreased the duration of rhinovirus infections, viral shedding, and the risk of rhinovirus illnesses in children with allergic asthma. ${ }^{19}$ The question on whether the same is true for coronavirus disease remained unanswered.

\section{Materials and Methods}

The authors performed a focused review on PubMed identifying the current data available on the management of severe asthma patients in the context of COVID-19. Case reports, prospective and retrospective studies and reviews concerning the treatment of severe asthma patients with COVID-19, published until June 2021, were included.

\section{Results \\ COVID-19, ACE2 and TMPRSS2}

\section{Expression and Asthma}

Angiotensin-converting enzyme 2 (ACE2) and transmembrane protease serine 2 (TMPRSS2) mediate SARS-CoV-2 infection of host cells. ${ }^{20}$ SARS-CoV-2 binds mainly to ACE2 receptors in host cells, which are abundant in the lungs, heart, blood vessels, and intestine. ${ }^{1,20}$ It has been suggested that ACE may be just one of several factors influencing the virus infection of cells, since not all cells that express ACE2 are susceptible to infection by SARSCoV-2, ${ }^{21}$ namely club cells, which do not get infected by this virus. ${ }^{22}$ Club cells have been reported to potentially express intrinsically high levels of some antiviral interferon (IFN)-stimulated genes, such as IFITMs (IFN-induced transmembrane proteins) and Ly6E (lymphocyte antigen $6 \mathrm{E}$ ), ${ }^{23}$ both described as coronavirus restriction factors. ${ }^{24,25}$

It has been questioned why children are less affected by SARS-CoV-2 infection even though they have similar seroprevalence rates. ${ }^{21}$ Some publications showed an association between levels of ACE2 expression in nasal epithelium and age, ${ }^{26}$ but in other studies, that was not found. ${ }^{27}$

Peters et al studied the relationship between demographic features and sputum ACE2 and TMPRSS2 gene expression in asthma by analyzing sputum cells from 330 participants in SARP-3 (Severe Asthma Research Program3) and 79 healthy control subjects. They found that expression levels of ACE2 and TMPRSS2 were similar between subjects with asthma and healthy subjects. Among severe asthma patients, diabetes mellitus, male sex, and African American race were associated with higher expression of ACE2 and TMPRSS2, as was the use of inhaled corticosteroids (ICS). However, they found no difference in the expression of these genes before and after treatment with intramuscular triamcinolone acetonide. ${ }^{28}$

\section{Asthma Phenotypes/Atopic Disease and COVID-19}

A meta-analysis of 131 studies and 410,382 patients found that COVID-19 asthmatic patients had a lower risk of death compared to non-asthmatic patients. ${ }^{10}$ Accordingly, other large studies and meta-analyses suggested that 
asthma patients may have a lower risk of severe COVID19. ${ }^{1,9,29}$ This could be related to the effect of atopy and type 2 inflammation in allergic patients. ${ }^{30}$ An Italian study found a decreased risk of severe COVID-19 disease in patients with atopic disease. ${ }^{31}$ However, in another study, there was no difference between the atopic and non-atopic groups in terms of intensive care unit (ICU) admission, duration of the hospitalization or mortality, suggesting that atopy was unrelated to COVID-19 severity. ${ }^{32}$

Different phenotypes of asthma may be associated with different COVID-19 outcomes. ${ }^{1,9,10}$ In particular, type 2 inflammation, mostly associated with asthma in the pediatric population or initiated during childhood, is characterized by a Th2 cells dominance and their hallmark cytokines IL-4, IL-5 and IL-13, together with eosinophilia. It has been shown that IL-13 downregulates the expression of ACE2 airway epithelial cells. Reduced levels of ACE2 transcripts have been associated with allergy, allergen exposure, and high IgE levels, ${ }^{33,34}$ as well as to higher levels of fractional exhaled nitric oxide. ${ }^{35}$ Eosinophilia has been negatively associated with COVID-19 susceptibility, whereas eosinopenia is a biomarker of severe COVID-19. Eosinophils express toll-like receptor 7, which recognizes single-stranded RNA, triggering an activation pathway that enhances the antiviral effects of eosinophils. ${ }^{36}$ Mast cells, also allergy-associated effector cells, have high antiviral potential owing to their production of interferons and other antiviral mediators. ${ }^{30}$

In contrast, the mechanisms associated with non-type 2 asthma and chronic obstructive pulmonary disease (COPD) (Th1-type or type 1 cytotoxic $\mathrm{T}$ (Tc1)-type responses and Th17 cell activation) may predispose to a more severe course of the disease: ${ }^{30}$

- Th1 and Th17 are associated with an increased expression of ACE2, although the underlying mechanisms are not fully understood. ${ }^{33,35,37}$

- Patients with non-type 2 asthma or COPD commonly suffer from other comorbidities that may predispose them to a more severe course of COVID-19 disease. $^{18}$

\section{Systemic and Inhaled Corticosteroids Use and COVID-19}

In hospitalized COVID-19 patients, the use of systemic dexamethasone resulted in lower 28-day mortality among those who were receiving either invasive mechanical ventilation or oxygen alone at randomization but not among those receiving no respiratory support. ${ }^{38}$ Using the OpenSAFELY platform, Williamson et al analyzed asthmatic patients' outcomes during COVID-19. The authors reported an increased risk of death in asthma patients with recent use of an oral corticosteroid (OCS). ${ }^{8}$ A study by Adir et al of COVID-19 asthma patients using the computerized database of Clalit Health Services also found that recent (within the previous 120 days) and chronic use of systemic corticosteroid in asthma patients was associated with a higher risk of moderate-to-severe COVID-19, all-cause mortality, and the composite endpoint of moderate-to-severe COVID-19 or all-cause mortality. ${ }^{39}$

The risk of getting COVID-19 in asthmatics taking OCS has also been analyzed. Hanon et al conducted an observational study using data from the Belgian Severe Asthma Registry. Of the 676 severe asthma patients surveyed, 58 were on maintenance OCS, none of whom was diagnosed with SARS-CoV-2 infection. ${ }^{40}$ A study of 1365 patients from the UK Severe Asthma Registry found that maintenance treatment with OCS was not associated with COVID-19. ${ }^{41}$

Regarding the use of ICS, Schultze et al reported an increased risk of COVID-19 related death among people with asthma prescribed high-dose ICS compared to those prescribed only short-acting beta-agonists (SABA) and those on low- or medium-dose ICS. However, quantitative bias analysis confirmed that a hypothetical unmeasured confounder of moderate strength could explain the observed results rather than these representing a harmful effect of ICS. ${ }^{42}$ In a large cohort of hospital admissions for COVID-19 across England, Scotland, and Wales, Bloom et al described different outcomes in patients with asthma or chronic pulmonary disease, according to whether or not these patients used ICS in the 2 weeks before hospital admission. ${ }^{43}$ Asthma patients aged 50 years and older who were using ICS had a $14 \%$ reduction in mortality risk compared to patients with no underlying respiratory condition. It was interesting to note that the proportion of people with asthma admitted to hospital with COVID-19 in this study $(8.6 \%$ aged $<16$ years, $20.9 \%$ aged $16-49$ years and $12.2 \%$ aged $\geq 50$ years) was higher than the national prevalence of asthma (approximately 7\% for each age group). Patients with underlying respiratory conditions were more likely to present with dyspnea and cough, but wheeze was uncommon except for pediatric asthma patients ( $<16$ years). In this group, no deaths 
occurred, but 10 patients (6.6\%) required admission to the ICU. Overall, patients with asthma aged 16-49 years and those aged 50 years and older were significantly more likely to receive critical care, non-invasive ventilation, and oxygen than non-asthmatic patients after adjusting for confounding factors. In these age groups, severe asthma patients had a significant increase in mortality compared to non-asthmatic patients. This increased risk was not observed in the other asthma patients.

Armentia et al conducted an observational study of 134 nursing home residents and 139 patients with uncontrolled asthma. The latter were all treated with ICS. Of the 139 asthma patients, 13 contracted COVID-19, none of whom became uncontrolled. Of the 134 nursing home residents, 80 were infected. Of these, $43 \%$ had previously received ICS for several health problems. Of the latter, $80 \%$ were asymptomatic, compared with $14 \%$ of deaths and $46 \%$ of severe disease in nursing home patients not on ICS. In the two study groups, prior allergic sensitization was associated with a good prognosis. The authors also found a significant difference in SARS-CoV-2 infections between asthma patients treated with ICS and nursing home residents not treated with ICS. ${ }^{29}$

A big-data retrospective study that analyzed 71,182 patients with asthma showed that the proportion of patients who used ICS was significantly reduced in asthmatics who required hospitalization due to COVID-19. ${ }^{44}$

Other authors found no difference between high-dose and lower dose of ICS in the risk of being infected. However, they found that hospitalized patients were on lower doses of ICS compared to ambulatory patients. ${ }^{41}$

\section{Biologic Therapies}

Table 1 summarizes published studies regarding the outcomes of COVID-19 infected asthma patients treated with biologics. Using an artificial intelligence platform, Izquierdo et al examined a total of 71,182 asthmatics patients' electronic health records and identified 865 patients $(1.21 \%)$ on biologic treatment. A total of 1006 patients were infected with COVID-19; 20 patients were being treated with a biologic. Two patients were hospitalized, both on treatment with mepolizumab, and one had a fatal outcome, a 52-year-old man with hypertension, diabetes mellitus and dyslipidemia. ${ }^{44}$

Rial et al studied 545 asthmatic patients on biologic treatment, 35 of whom were infected with SARS-CoV-2. Among eight hospital-admitted patients, one needed intensive care and one died because of COVID-19, an 82- year-old patient with hypertension, diabetes and ischemic cardiopathy. Comparing the different treatment groups, the authors found a higher probability, but not significant, of COVID-19 infection in patients treated with reslizumab. The authors also grouped the patients treated with the three anti-IL5 biologic drugs (mepolizumab, reslizumab and benralizumab) and compared with the ones under omalizumab: no statistical difference was observed regarding severity of the disease, presence of comorbidities, ICU admissions or mortality. The same result was obtained when comparing patients in this study and a cohort of patients with mild and moderate asthma with no biological treatment. $^{45}$

In an Italian study, which included 473 severe asthma patients on ongoing treatment with a biologic, the prevalence of confirmed SARS-CoV-2 infection was of $0.846 \%$ and no difference was observed in comparison to data in the geography-matched general population, which presented a prevalence of $0.8 \%{ }^{46}$

Similar results were found in a Belgium study that included 676 severe asthma patients, 434 of these on a biological drug. Fourteen patients were infected with SARS-CoV-2 and 11 of these were under biologic treatment (4 with omalizumab and 7 with an anti-IL5 or antiIL5R therapy). Five patients were hospitalized (one with no biologic treatment and four under anti-IL5/-IL5R treatment) but there was no need of non-invasive or invasive ventilation or ICU admission in either group of patients. There were also no reported deaths. Despite the low number of confirmed cases, the authors found no difference in the incidence of COVID-19 between patients treated with biologics and patients not receiving any biologic treatment. ${ }^{40}$ The study was unpowered to discriminate any differences regarding distinct biologic treatments.

A study in the population of the Severe Asthma Network in Italy (SANI) and in an additional Italian Centre included 1504 patients, $65 \%$ of whom were treated with biologics. The authors reported 26 confirmed or highly suspected COVID-19 cases, 21 of whom were on biological treatment. Four patients were hospitalized and one of them was admitted to the ICU. Two patients died, which accounted for a COVID-19 related mortality of $7.7 \%$, lower than in the general Italian population $(14.5 \%) .{ }^{47}$

Another Italian study involving 41 centers of the Italian Registry of Severe Asthma (IRSA) network included 558 patients, 7 of whom contracted COVID-19. Two of them were hospitalized, but none was admitted to the ICU. 


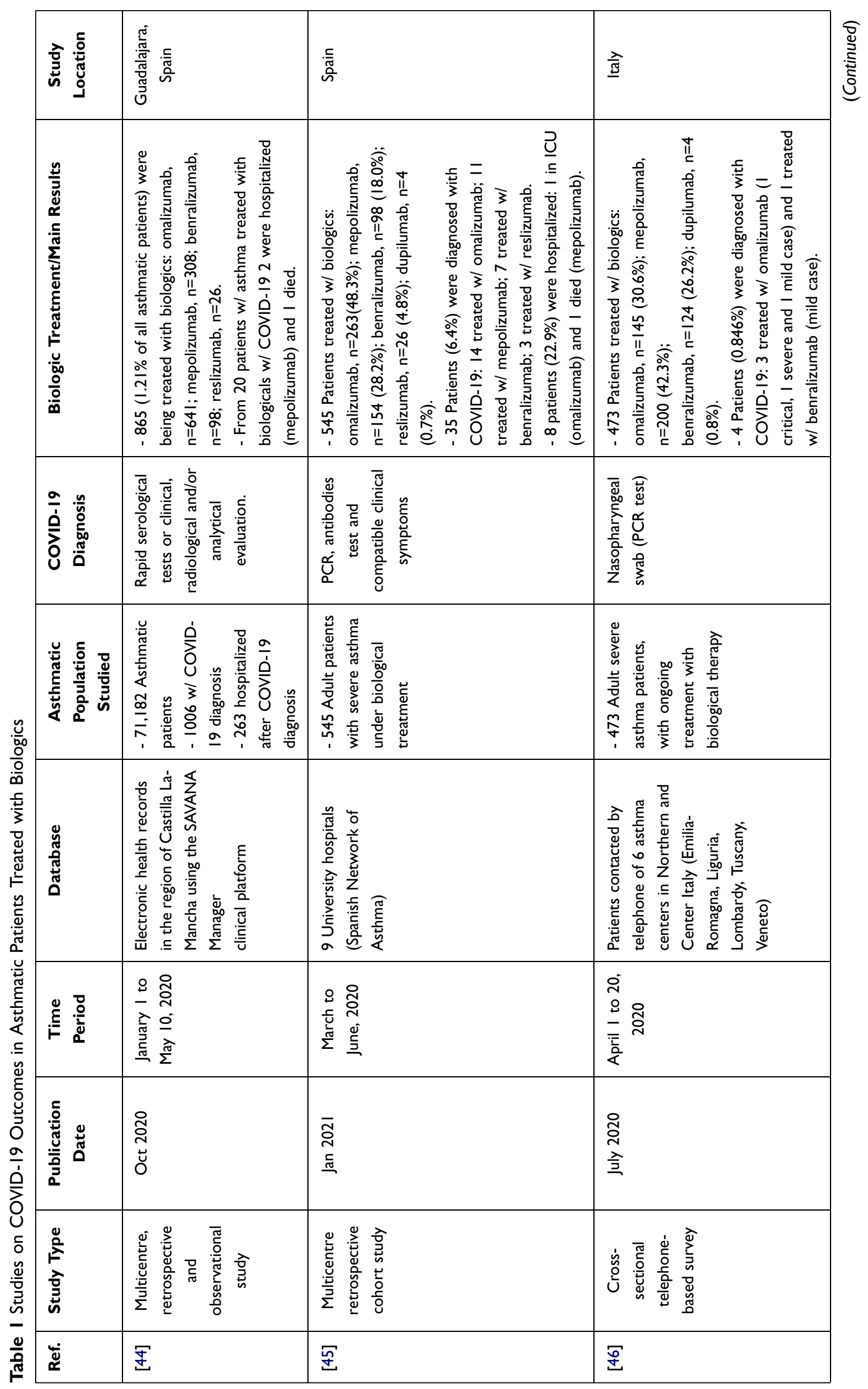




\begin{tabular}{|c|c|c|c|c|}
\hline 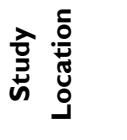 & 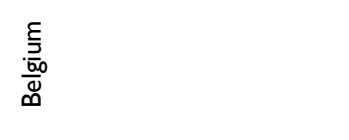 & 첲 & 첲 & 弚 \\
\hline 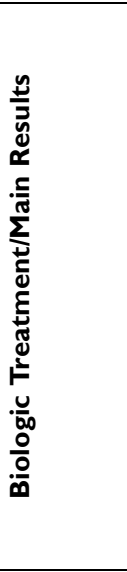 & 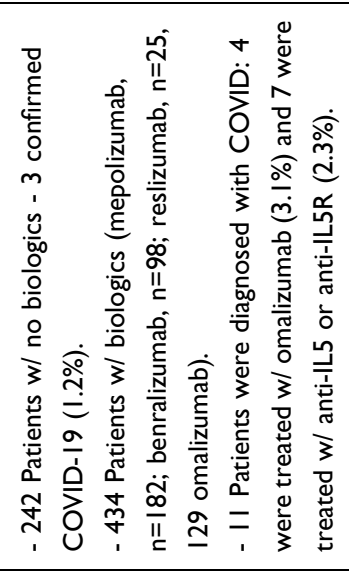 & 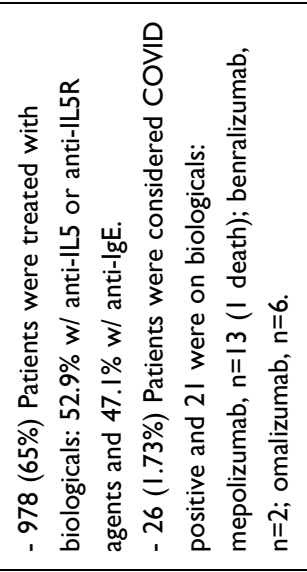 & 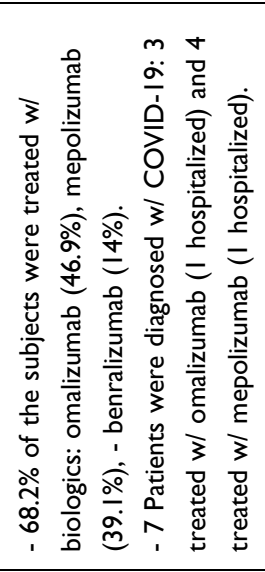 & 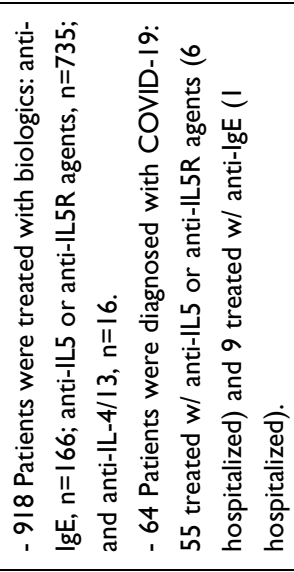 \\
\hline 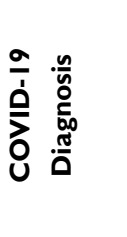 & 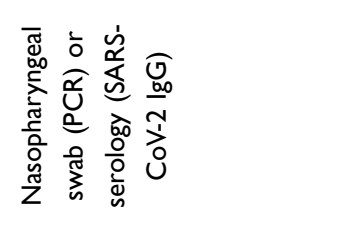 & 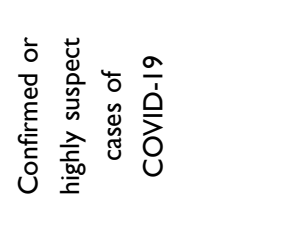 & 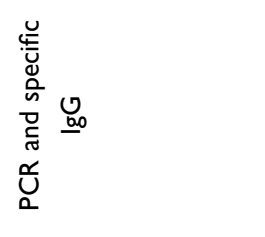 & 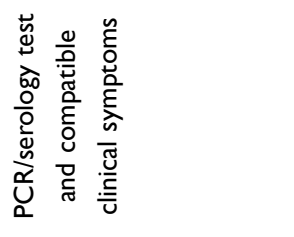 \\
\hline 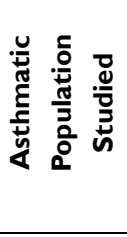 & 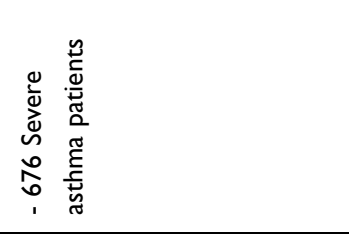 & 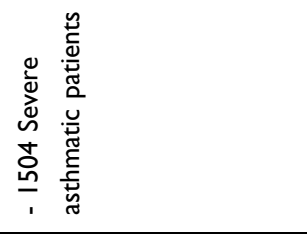 & 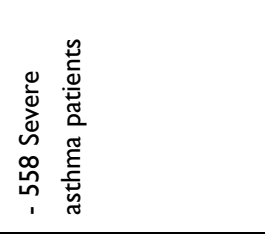 & 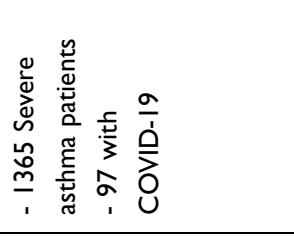 \\
\hline $\begin{array}{l}0 \\
\text { हूँ } \\
\text { हैँ } \\
\text { हूँ }\end{array}$ & 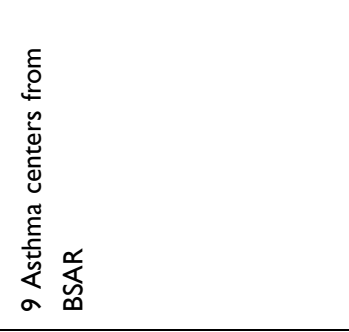 & 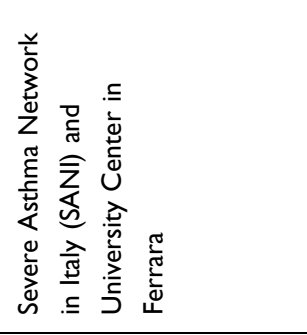 & 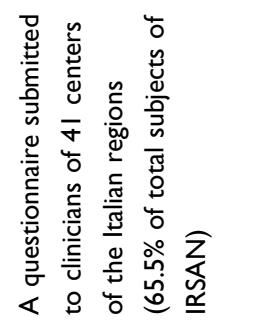 & 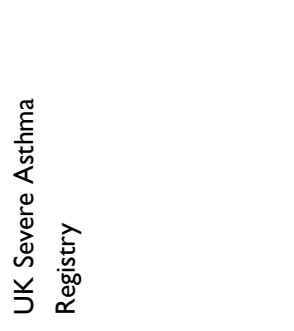 \\
\hline 号 & 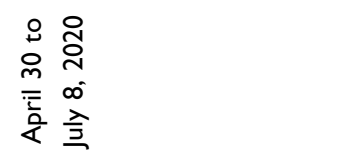 & & 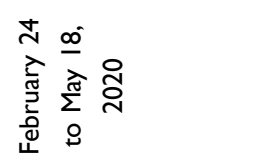 & 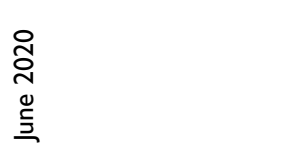 \\
\hline 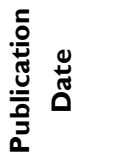 & 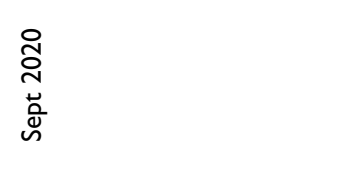 & 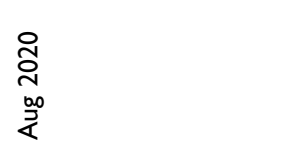 & 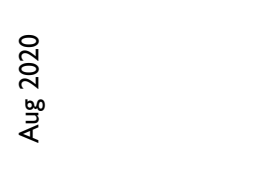 & 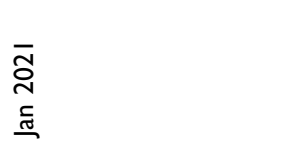 \\
\hline 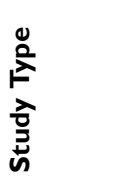 & 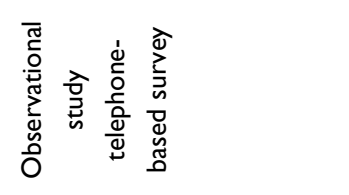 & 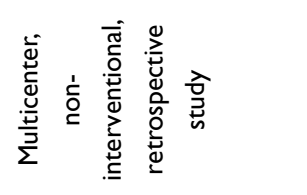 & 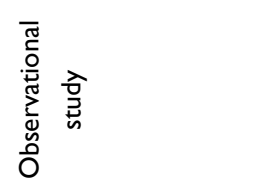 & 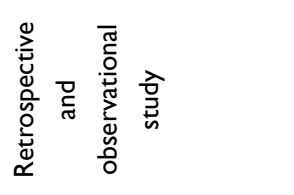 \\
\hline $\begin{array}{l}\dot{\Phi} \\
\check{\varpi}\end{array}$ & 宇 & 胥 & 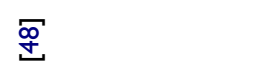 & $\bar{\Xi}$ \\
\hline
\end{tabular}




\begin{tabular}{|c|c|c|c|c|c|}
\hline $\bar{\Phi} \frac{\bar{g}}{\underline{\underline{m}}}$ & 를 & 氒 & 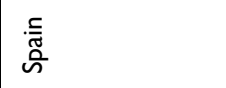 & 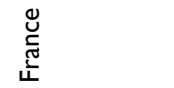 & 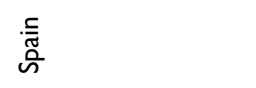 \\
\hline 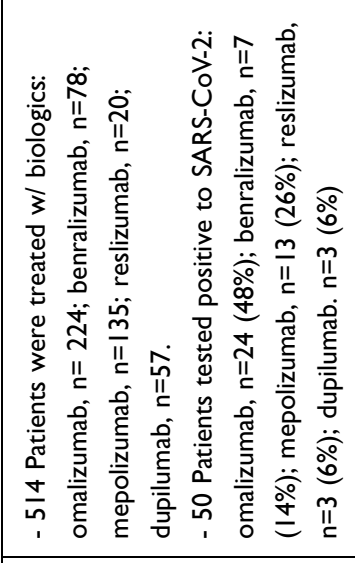 & 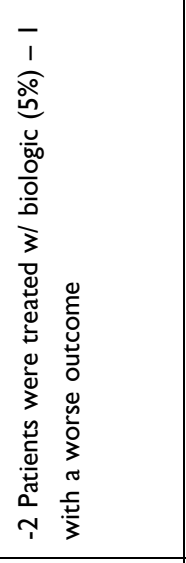 & 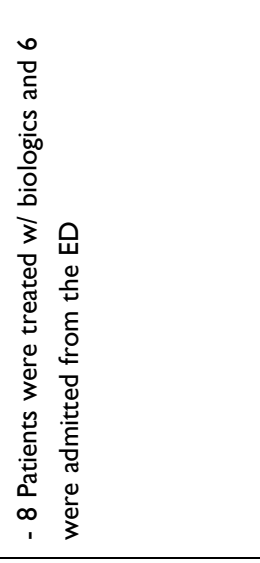 & 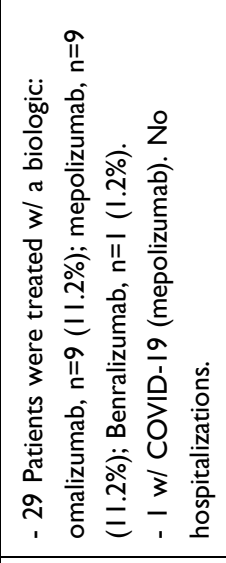 & 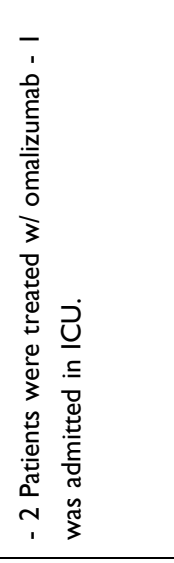 & 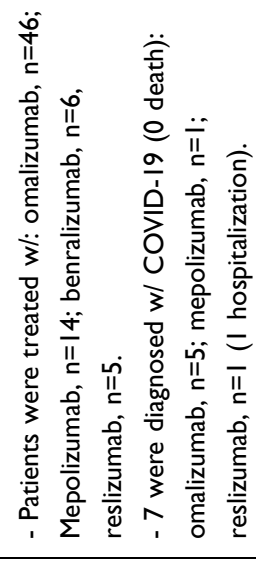 \\
\hline 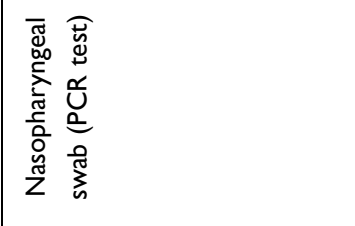 & & 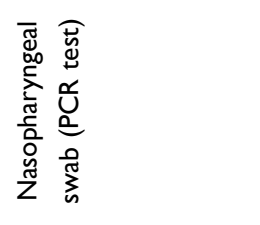 & 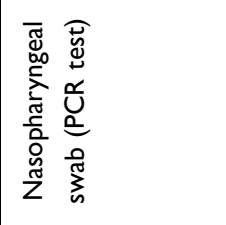 & 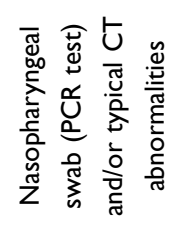 & 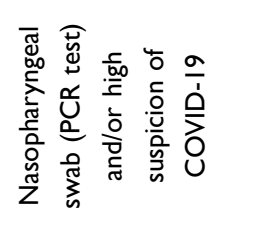 \\
\hline 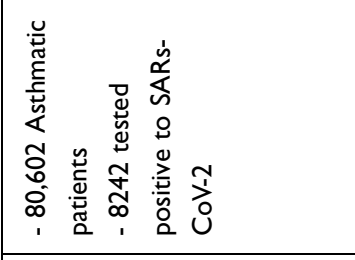 & 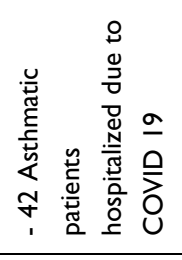 & 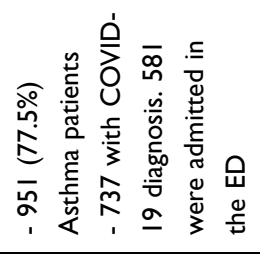 & 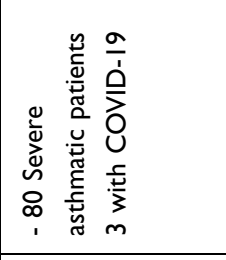 & 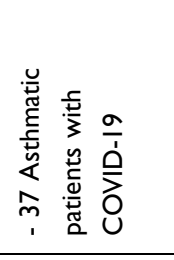 & 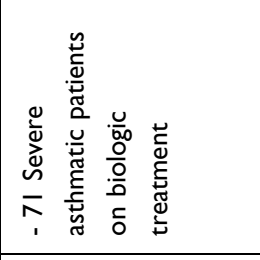 \\
\hline 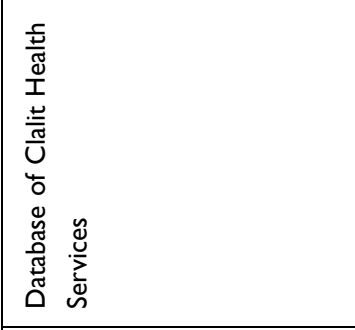 & 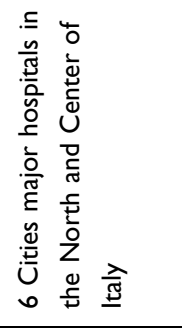 & 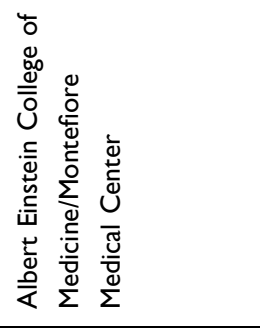 & 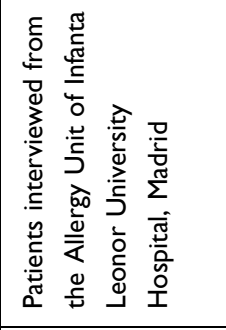 & 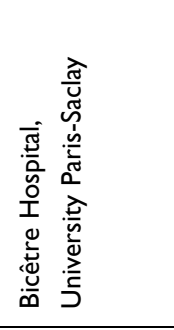 & 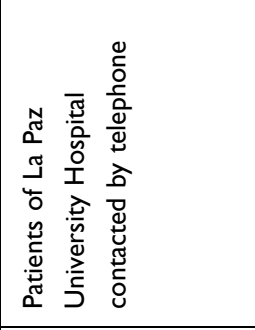 \\
\hline 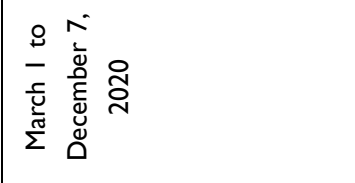 & 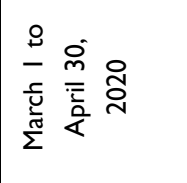 & 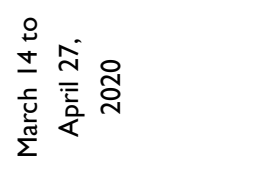 & 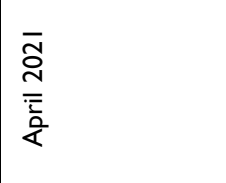 & 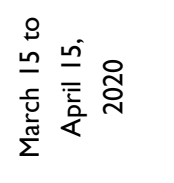 & \\
\hline 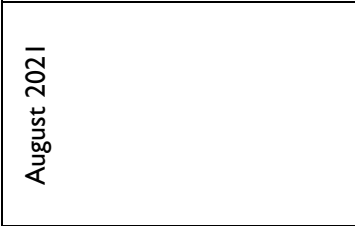 & 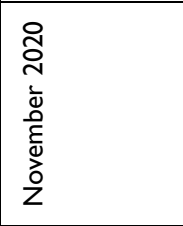 & 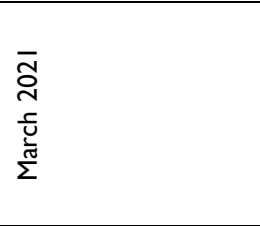 & 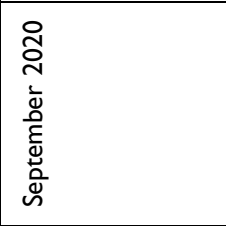 & 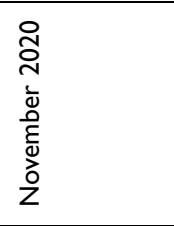 & 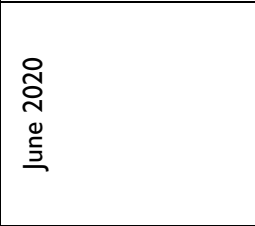 \\
\hline 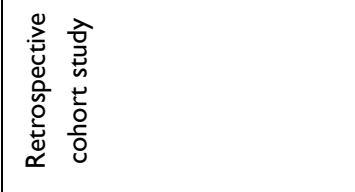 & 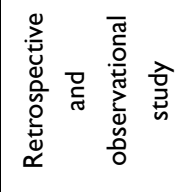 & 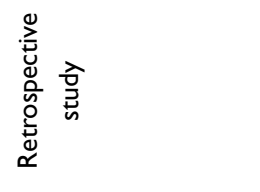 & 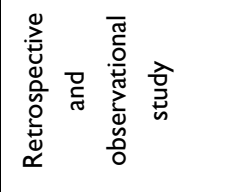 & 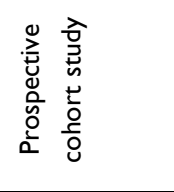 & 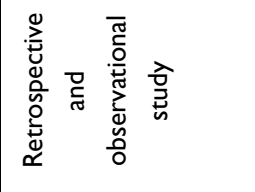 \\
\hline 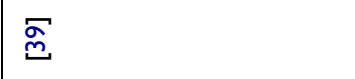 & 㝋 & 它 & $\overline{\tilde{n}}$ & $\sqrt[\widetilde{n}]{2}$ & $\overline{\tilde{n}}$ \\
\hline
\end{tabular}




\begin{tabular}{|c|c|}
\hline 害高 & 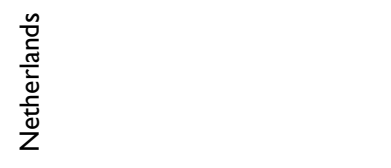 \\
\hline 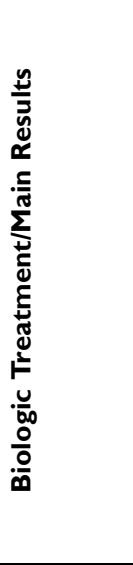 & 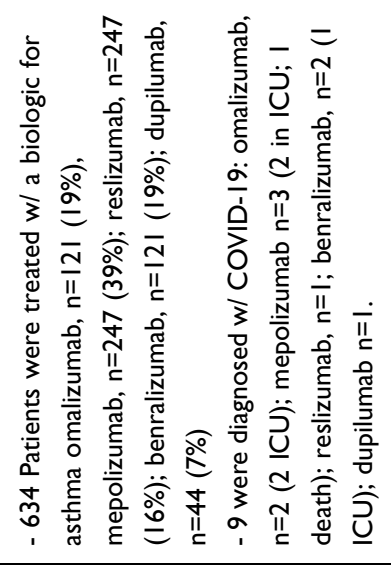 \\
\hline 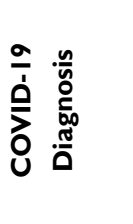 & 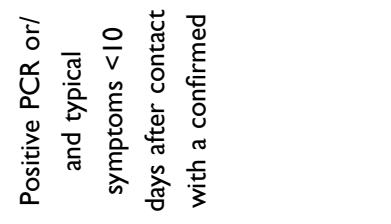 \\
\hline 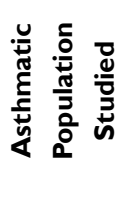 & 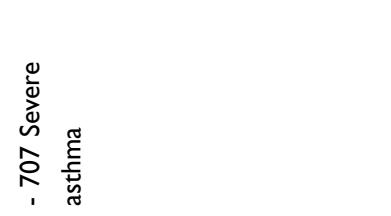 \\
\hline $\begin{array}{l}\text { ڤँ } \\
\text { हैँ } \\
\text { ปूँ }\end{array}$ & 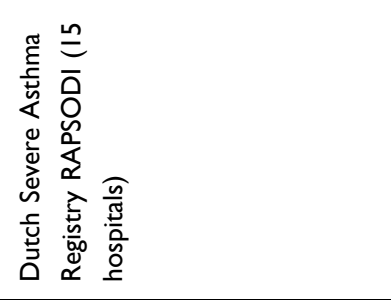 \\
\hline 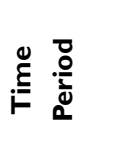 & 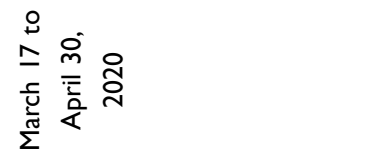 \\
\hline مَّ & 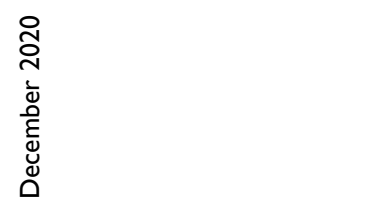 \\
\hline 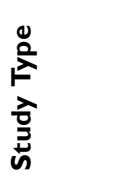 & 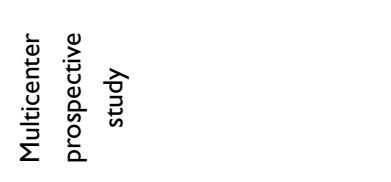 \\
\hline 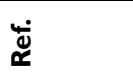 & 岧 \\
\hline
\end{tabular}

There were no reported deaths. The authors found no significant difference in COVID-19 frequency between severe asthma patients on biologics compared to those not on biologics. ${ }^{48}$

Smith et al studied 1365 patients from the UK Severe Asthma Registry, 97 of whom had suspected or confirmed COVID-19. Of the 1365 severe asthma patients, 918 were treated with biologics. The authors also found no association between biological treatment and the risk of COVID19. Neither did they find an association between the type of biologic and COVID-19. ${ }^{41}$

In the study by Adir et al of COVID-19 asthma patients, the authors found that biologics use was not associated with an increased risk of the studied endpoints: moderate-to-severe COVID-19, all-cause mortality and the composite endpoint of moderate-to-severe COVID-19 or all-cause mortality. ${ }^{39}$

Caminati et al reviewed the medical records of patients admitted to COVID-Units of six Italian cities' major hospitals. Of the 2000 COVID-19 patients, 42 (2.1\%) had asthma. Of these, $24 \%$ had severe asthma. Only $5 \%$ of asthma patients were treated with biologics. The authors found that GINA step 4-5 asthma was significantly associated with the probability of a worse outcome. ${ }^{49}$

In a study of 4558 COVID-19 patients of the Albert Einstein College of Medicine/Montefiore Medical Center, 951 patients had asthma, 8 of whom were on biologics. The authors did not find an association between treatment with biologics and the odds of being admitted from the emergency department. ${ }^{50}$

Haroun-Díaz et al reported on 80 severe asthma patients from the Infanta Leonor University Hospital, $23.7 \%$ of whom were treated with biologic agents. Of the total 80 patients, 3 had COVID-19, one of them being on mepolizumab. None of the patients required admission to the ICU and there were no reported deaths. ${ }^{51}$

Beurnier et al conducted a prospective cohort of COVID-19 asthma patients hospitalized in the Bicêtre Hospital, University Paris-Saclay, France. Of the total 768 COVID-19 patients, 37 were asthmatic. Eleven asthma patients were GINA step 5 and two of them were on omalizumab. Three patients died, none of whom were being treated with omalizumab. One of the patients on omalizumab required admission to the ICU. ${ }^{52}$

Domínguez-Ortega et al studied 71 severe asthma patients on therapy with biologics, most of them $(n=63)$ treated at home on a self-administration program. Of these, seven had a COVID-19 diagnosis. This rate of infection 
$(9.85 \%)$ was similar to that of the general population. Only one of the patients required hospitalization and all recovered. ${ }^{53}$

Eger et al conducted a prospective study on the Dutch Severe Asthma Registry RAPSODI (Registry of Adult Patients with Severe asthma for Optimal Disease management) population. Of the 707 patients included, 634 were treated with biologics, $9(1.42 \%)$ of whom were diagnosed with COVID-19. Seven of these nine patients were hospitalized and 5 of these were admitted to the ICU for mechanical ventilation, one (14\%) of whom died. The authors found that, compared to the general population, the RAPSODI population on biologics had a higher risk of being infected by SARS-CoV-2 and of COVID-19 related hospitalization, intubation and death. However, the authors suggested that this could be explained by the higher prevalence of obesity in biologic-treated patients. They also pointed out that, compared to other studies, the RAPSODI population had a higher proportion of patients who had adult-onset asthma, were non-atopic and had been previously on chronic OCS treatment. ${ }^{54}$

There were also a few published case reports. An asthmatic patient on omalizumab became infected by SARS-CoV-2 and had a mild disease course ${ }^{55}$; four severe asthma patients treated with benralizumab had COVID-19 and only one required hospitalization but recovered. ${ }^{56-58}$ Azim et al reported on another four patients on mepolizumab who had COVID-19, one of whom was hospitalized, but there were no deaths. ${ }^{59}$ Aksu et al also reported the case of a COVID-19 severe asthma patient on mepolizumab who had mild disease. ${ }^{60}$ Bhalla et al reported the case of a patient on dupilumab who had mild COVID-19. ${ }^{61}$

\section{Triple Therapy with ICS, LAMA, and LABA}

Triple therapy with ICS, LAMA (long-acting muscarinic antagonists) and LABA (long-acting beta2 agonists) has gained importance as a potential treatment for asthma patients. GINA guidelines recommend LAMA as add-on therapy in steps 4 and $5 .{ }^{62}$ Triple therapy has been reported to improve lung function and decrease exacerbations in uncontrolled asthma compared to treatment with ICS and LABA. ${ }^{63,64}$ There is limited evidence about the role of triple therapy in asthmatic patients in the context of SARS-CoV-2 infection. Liang et al reported the case of a 54-year-old man with asthma and allergic rhinitis who contracted COVID-19 and required admission to the ICU and tracheal intubation. After extubation and during systemic glucocorticoids treatment and ICS/LABA combination, the patient remained very symptomatic and was therefore treated with inhaled budesonide/glycopyrrolate/ formoterol fumarate, with a good response. This case suggested that triple therapy can be beneficial in SARSCoV-2-infected asthmatic patients. However, this is only a case report. Furthermore, this patient had a smoking history and emphysema visible in the computed tomography scan, which supports the diagnosis of COPD and therefore makes it difficult to interpret these results. ${ }^{65}$

It has been suggested, from a study with primary human nasal and tracheal epithelial cell cultures, that a combination of glycopyrronium, formoterol and budesonide inhibit Coronavirus 229E (HCoV-229E) replication and modulate infection-induced inflammation in the airway. It is possible that these drugs also have an effect against SARS-CoV-2 infection, although that has not been studied. ${ }^{66}$

\section{Discussion}

Despite some controversy, the reviewed evidence suggests that severe asthma patients do not have an increased risk of COVID-19 poor outcomes. It also suggests that it is safe to treat asthmatic patients with ICS and biologics during the COVID-19 pandemic, even though some studies pointed to high doses of ICS being detrimental. ${ }^{41,42}$ The chronic use of OCS might be associated with poor COVID-19 outcomes, ${ }^{39}$ although that is not consensual. There is very limited evidence concerning the use of triple therapy for asthma in the context of COVID-19. The latter is, so far, promising, but it is impossible at this point to take any definite conclusions on this matter. ${ }^{65}$

Issues regarding the ideal dose and timing of corticoids administration in the treatment of COVID-19 asthma patients remain to be clarified. Several randomized controlled trials are underway to address the role of ICS in treating COVID-19 once patients have become infected with SARS-CoV-2 (NCT04355637, NCT04331470, NCT04377711, NCT04330586, and NCT04416399). ${ }^{43}$

Several organizations, as the Global Initiative for Asthma (GINA), the National Institute for Health and Care Excellence (NICE), and the British Thoracic Society (BTS) recommend not stopping treatment with biologics in asthmatic patients during the COVID-19 pandemic. $^{62,67,68}$

Although Eger et al found that patients on biologics had an increased risk of COVID-19 and poor related 
outcomes, this study had some confounding factors, namely the higher prevalence of obesity in the population treated with biologics. ${ }^{54}$ Several studies on this subject refer as a methodological limitation the fact that in the beginning of the pandemic there was a restriction in the number of diagnostic tests available. The milder cases were most often quarantined and not tested. This may have resulted on a non-identification of many asthmatic patients with or without biological treatment and may bias the results. ${ }^{40,44,46,53,54}$ The number of asthma admissions particularly in children may also been influenced by the social distancing and the use of face masks. ${ }^{69}$

Although the self-administration option of biologics treatment was not mentioned on most of the presented studies, Domínguez-Ortega et $\mathrm{al}^{53}$ referred that the majority of patients on biologics (89\%) were treated at home. Selfadministration of biologic treatments should be increasingly available and encouraged, as it decreases the need of hospital visits which could be beneficial in the present pandemic situation, avoiding withholding the medication.

There are still many doubts surrounding this topic, namely regarding the effects of asthma phenotypes on COVID-19. It has been suggested that T2-low patients with asthma may have a higher risk for COVID-19 severe outcomes. ${ }^{50}$

\section{Conclusion}

Current evidence supports that severe asthma patients should maintain their medication during the COVID-19 pandemic, including biologic agents. It is recommended that, along with all the efforts to avoid exposure to the SARS-CoV-2 virus together with COVID-19 vaccination, regular asthma treatments should be optimized to maintain asthma control. ${ }^{6,62,70}$ In particular, the evidence suggests that ICS and biologics do not increase the risk of SARS-CoV-2 infection or of poor related outcomes, while doubts remain about dosing and timing. The evidence regarding OCS is contradictory and that on triple therapy in this context is insufficient.

More studies are needed to address the role of asthma medications and asthma's different phenotypes on the frequency and course of COVID-19.

\section{Disclosure}

The authors report no conflicts on interest in this work.

\section{References}

1. Morais-Almeida M, Pité H, Aguiar R, et al. Asthma and the coronavirus disease 2019 pandemic: a literature review. Int Arch Allergy Immunol. 2020;181(9):680-688. doi:10.1159/000509057
2. Lupia T, Scabini S, Mornese Pinna S, Di Perri G, De Rosa FG, Corcione S. 2019 novel coronavirus (2019-nCoV) outbreak: a new challenge. J Glob Antimicrob Resist. 2020;21:22-27. doi:10.1016/j. jgar.2020.02.021

3. Yang J, Zheng Y, Gou X, et al. Prevalence of comorbidities and its effects in patients infected with SARS-CoV-2: a systematic review and meta-analysis. Int $J$ Infect Dis. 2020;94:91-95. doi:10.1016/j. ijid.2020.03.017

4. Fang L, Karakiulakis G, Roth M. Are patients with hypertension and diabetes mellitus at increased risk for COVID-19 infection? Lancet Respir Med. 2020;8(4):e21. doi:10.1016/S2213-2600(20)30116-8

5. Skevaki C, Karsonova A, Karaulov A, Xie M, Renz H. Asthmaassociated risk for COVID-19 development. J Allergy Clin Immunol. 2020;146(6):1295-1301. doi:10.1016/j.jaci.2020.09.017

6. Centers for Disease Control and Prevention. People with moderate to severe asthma. Available from: https://www.cdc.gov/coronavirus/2019ncov/need-extra-precautions/asthma.html. Accessed April 32021.

7. Yang JM, Koh HY, Moon SY, et al. Allergic disorders and susceptibility to and severity of COVID-19: a nationwide cohort study. J Allergy Clin Immunol. 2020;146(4):790-798. doi:10.1016/j.jaci.2020.08.008

8. Williamson EJ, Walker AJ, Bhaskaran K, et al. Factors associated with COVID-19-related death using OpenSAFELY. Nature. 2020;584 (7821):430-436. doi:10.1038/s41586-020-2521-4

9. Patrucco F, Villa E, Foci V, Benfante A, Bellocchia M, Solidoro P. Severe asthma at COVID-19 time: what is new on biologic therapies. Minerva Med. 2021;112(1):114-117. doi:10.23736/s0026-4806.20.06727-0

10. Liu S, Cao Y, Du T, Zhi Y. Prevalence of comorbid asthma and related outcomes in COVID-19: a systematic review and meta-analysis. J Allergy Clin Immunol Pract. 2021;9(2):693-701. doi:10.1016/j.jaip.2020.11.054

11. Mahdavinia M, Foster KJ, Jauregui E, et al. Asthma prolongs intubation in COVID-19. J Allergy Clin Immunol Pract. 2020;8 (7):2388-2391. doi:10.1016/j.jaip.2020.05.006

12. Rosenthal JA, Awan SF, Fintzi J, Keswani A, Ein D. Asthma is associated with increased risk of intubation but not hospitalization or death in coronavirus disease 2019. Ann Allergy Asthma Immunol. 2021;126(1):93-95. doi:10.1016/j.anai.2020.10.002

13. Chhiba KD, Patel GB, Vu THT, et al. Prevalence and characterization of asthma in hospitalized and nonhospitalized patients with COVID-19. J Allergy Clin Immunol. 2020;146(2):307-314.e4. doi:10.1016/j.jaci.2020.06.010

14. Akenroye AT, Wood R, Keet C. Asthma, biologics, corticosteroids, and coronavirus disease 2019. Ann Allergy Asthma Immunol. 2020;125:2019-2020. doi:10.1016/j.anai.2020.05.001

15. Morais-Almeida M, Aguiar R, Martin B, et al. COVID-19, asthma, and biological therapies: what we need to know. World Allergy Organ J. 2020;13(5):100126. doi:10.1016/j.waojou.2020.100126

16. Chung KF, Wenzel SE, Brozek JL, et al. International ERS/ATS guidelines on definition, evaluation and treatment of severe asthma. Eur Respir J. 2014;43(2):343-373. doi:10.1183/09031936.00202013

17. Xia Z. Eosinopenia as an early diagnostic marker of COVID-19 at the time of the epidemic. EClinicalMedicine. 2020;23:19-20. doi:10. 1016/j.eclinm.2020.100398

18. Peters MC, McGrath KW, Hawkins GA, et al. Plasma interleukin-6 concentrations, metabolic dysfunction, and asthma severity: a cross-sectional analysis of two cohorts. Lancet Respir Med. 2016;4 (7):574-584. Erratum in: Lancet Respir Med. 2018 Mar;6(3):e10. doi:10.1016/S2213-2600(16)30048-0

19. Esquivel A, Busse WW, Calatroni A, et al. Effects of omalizumab on rhinovirus infections, illnesses, and exacerbations of asthma. Am J Respir Crit Care Med. 2017;196(8):985-992. doi:10.1164/rccm. 201701-0120OC

20. Hoffmann M, Kleine-Weber H, Schroeder S, et al. SARS-CoV-2 cell entry depends on ACE2 and TMPRSS2 and is blocked by a clinically proven protease inhibitor. Cell. 2020;181(2):271-280.e8. doi:10. 1016/j.cell.2020.02.052 
21. Sanchez-David RY, Swann OC, Peacock TP, Barclay WS. ACE2: the only thing that matters? Am J Respir Crit Care Med. 2020;202 (2):161-163. doi:10.1164/rccm.202006-2151ED

22. Hou YJ, Okuda K, Edwards CE, et al. SARS-CoV-2 reverse genetics reveals a variable infection gradient in the respiratory tract. Cell. 2020;182(2):429-446.e14. doi:10.1016/j.cell.2020.05.042

23. Wu X, Dao Thi VL, Huang Y, et al. Intrinsic immunity shapes viral resistance of stem cells. Cell. 2018;172(3):423-420438.e25. doi:10.1016/j.cell.2017.11.018

24. Pfaender S, Mar KB, Michailidis E, et al. LY6E impairs coronavirus fusion and confers immune control of viral disease. Nat Microbiol. 2020;5(11):1330-1339. doi:10.1038/s41564-020-0769-y

25. Huang IC, Bailey CC, Weyer JL, et al. Distinct patterns of IFITM-mediated restriction of filoviruses, SARS coronavirus, and influenza A virus. PLoS Pathog. 2011;7(1):e1001258. doi:10.1371/ journal.ppat. 1001258

26. Bunyavanich S, Do A, Vicencio A. Nasal gene expression of angiotensin-converting enzyme 2 in children and adults. JAMA. 2020;323(23):2427-2429. doi:10.1001/jama.2020.8707

27. Lee IT, Nakayama T, Wu CT, et al. Robust ACE2 protein expression localizes to the motile cilia of the respiratory tract epithelia and is not increased by ACE inhibitors or angiotensin receptor blockers. medRxiv. 2020;2020. Update in: Nat Commun. 2020 Oct 28; 11(1):5453. doi:10.1101/2020.05.08.20092866

28. Peters MC, Sajuthi S, Deford P, et al. COVID-19-related genes in sputum cells in asthma: relationship to demographic features and corticosteroids. Am J Respir Crit Care Med. 2020;202(1):83-90. doi:10.1164/rccm.202003-08210C

29. Armentia A, Cortés SF, Simón AM, et al. Inhaled corticosteroids may have a protective effect against coronavirus infection. Allergol Immunopathol. 2021;49(1):113-117. doi:10.15586/aei.v49i1.40

30. Skevaki C, Karsonova A, Karaulov A, et al. SARS-CoV-2 infection and COVID-19 in asthmatics: a complex relationship. Nat Rev Immunol. 2021:0123456789. doi:10.1038/s41577-021-00516-z

31. Scala E, Abeni D, Tedeschi A, et al. Atopic status protects from severe complications of COVID-19. Allergy. 2021;76(3):899370902. doi:10.1111/all.14551

32. Timberlake DT, Narayanan D, Ogbogu PU, et al. Severity of COVID-19 in hospitalized patients with and without atopic disease. World Allergy Organ J. 2021;14(2):100508. doi:10.1016/j.waojou.2021.100508

33. Jackson DJ, Busse WW, Bacharier LB, et al. Association of respiratory allergy, asthma, and expression of the SARS-CoV-2 receptor ACE2. J Allergy Clin Immunol. 2020;146(1):203-206.e3. doi:10.1016/j.jaci. 2020.04.009

34. Kimura H, Francisco D, Conway M, et al. Type 2 inflammation modulates ACE2 and TMPRSS2 in airway epithelial cells. J Allergy Clin Immunol. 2020;146(1):80-88.e8. doi:10.1016/j.jaci.2020.05.004

35. Bradding P, Richardson M, Hinks TSC, et al. ACE2, TMPRSS2, and furin gene expression in the airways of people with asthma-implications for COVID-19. J Allergy Clin Immunol. 2020;146(1):208-211. doi:10.1016/j.jaci.2020.05.013

36. Phipps S, Lam CE, Mahalingam S, et al. Eosinophils contribute to innate antiviral immunity and promote clearance of respiratory syncytial virus. Blood. 2007;110(5):1578-1586. doi:10.1182/blood-2007-01-071340

37. Peters MC, Sajuthi S, Deford P, et al. COVID-19-related genes in sputum cells in asthma. relationship to demographic features and corticosteroids. Am J Respir Crit Care Med. 2020;202:83-90. Erratum in: 440 Am J Respir Crit Care Med. 2020 Dec 15; 202(12):1744-1746. doi:10.1164/rccm.202003-0821OC

38. The RECOVERY Collaborative Group. Dexamethasone in hospitalized patients with Covid-19. N Engl J Med. 2021;384(8):693-704. doi:10.1056/NEJMoa2021436

39. Adir Y, Humbert M, Saliba W. COVID-19 risk and outcomes in adult asthmatic patients treated with biologics or systemic corticosteroids: nationwide real-world evidence. J Allergy Clin Immunol. 2021;148 (2):S0091-6749(21)00938-6. doi:10.1016/j.jaci.2021.06.006
40. Hanon S, Brusselle G, Deschampheleire M, et al. COVID-19 and biologics in severe asthma: data from the Belgian severe asthma registry. Eur Respir J. 2020;56(6):56-59. doi:10.1183/13993003.02857-2020

41. Smith SJ, Busby J, Heaney LG, et al. The impact of the first COVID-19 surge on severe asthma patients in the UK. Which is worse: the virus or the lockdown? ERJ Open Res. 2021;7 (1):00768-2020. doi:10.1183/23120541.00768-2020

42. Schultze A, Walker AJ, MacKenna B, et al. Risk of COVID-19related death among patients with chronic obstructive pulmonary disease or asthma prescribed inhaled corticosteroids: an observational cohort study using the OpenSAFELY platform. Lancet Respir Med. 2020;8(11):1106-1120. doi:10.1016/S2213-2600(20)30415-X

43. Bloom C, Tom D, Docherty A, et al. Risk of adverse outcomes in patients with underlying respiratory conditions hospitalised with COVID-19 using the ISARIC WHO clinical characterisation protocol: a national, multicentre prospective cohort. Lancet Respir Med. 2021;9(7):699-711. doi:10.1016/S2213-2600(21)00013-8

44. Izquierdo JL, Almonacid C, González Y, et al. The impact of COVID-19 on patients with asthma. Eur Respir J. 2020:2003142. doi:10.1183/13993003.03142-2020

45. Rial MJ, Valverde M, Del Pozo V, et al. Clinical characteristics in 545 patients with severe asthma on biological treatment during the COVID-19 outbreak. J Allergy Clin Immunol Pract. 2021;9(1):487489.e1. doi:10.1016/j.jaip.2020.09.050

46. Matucci A, Caminati M, Vivarelli E, et al. COVID-19 in severe asthmatic patients during ongoing treatment with biologicals targeting type 2 inflammation: results from a multicenter Italian survey. Allergy Eur J Allergy Clin Immunol. 2020:871-874. doi:10.1111/ all.14516

47. Heffler E, Detoraki A, Contoli M, et al. COVID-19 in Severe Asthma Network in Italy (SANI) patients: clinical features, impact of comorbidities and treatments. Allergy Eur $J$ Allergy Clin Immunol. 2020;2:887-892. doi:10.1111/all.14532

48. Antonicelli L, Tontini C, Manzotti G, et al. Severe asthma in adults does not significantly affect the outcome of COVID-19 disease: results from the Italian severe asthma registry. Allergy. 2021;76 (3):902-905. doi:10.1111/all.14558

49. Caminati M, Vultaggio A, Matucci A, et al. Asthma in a large COVID-19 cohort: prevalence, features, and determinants of COVID-19 disease severity. Respir Med. 2021;176:106261. doi:10. 1016/j.rmed.2020.106261

50. Ferastraoaru D, Hudes G, Jerschow E, et al. Eosinophilia in asthma patients is protective against severe COVID-19 illness. $J$ Allergy Clin Immunol Pract. 2021;9(3):1152-1162.e3. doi:10.1016/j.jaip.2020.12.045

51. Haroun-Díaz E, Vázquez de la Torre M, Ruano FJ, et al. Severe asthma during the COVID-19 pandemic: clinical observations. J Allergy Clin Immunol Pract. 2020;8(8):2787-2789. doi:10.1016/j.jaip.2020.06.033

52. Beurnier A, Jutant EM, Jevnikar M, et al. Characteristics and outcomes of asthmatic patients with COVID-19 pneumonia who require hospitalisation. Eur Respir J. 2020;56(5):2001875. doi:10.1183/ 13993003.01875-2020

53. Domínguez-Ortega J, López-Carrasco V, Barranco P, et al. Early experiences of SARS-CoV-2 infection in severe asthmatics receiving biologic therapy. J Allergy Clin Immunol Pract. 2020;8(8):27 84-2786. doi:10.1016/j.jaip.2020.06.027

54. Eger K, Hashimoto S, Braunstahl GJ, et al. Poor outcome of SARS-CoV-2 infection in patients with severe asthma on biologic therapy. Respir Med. 2021;177(December2020):106287. doi:10.1016/ j.rmed.2020.106287

55. Lommatzsch M, Stoll P, Virchow JC. COVID-19 in a patient with severe asthma treated with omalizumab. Allergy Eur J Allergy Clin Immunol. 2020;75(10):2705-2708. doi:10.1111/all.14456

56. García-Moguel I, Díaz Campos R, Alonso Charterina S, Fernández Rodríguez C, Fernández Crespo J. COVID-19, severe asthma, and biologics. Ann Allergy Asthma Immunol. 2020;125(3):357-359.e1. doi:10.1016/j.anai.2020.06.012 
57. Renner A, Marth K, Patocka K, Pohl W. COVID-19 in a severe eosinophilic asthmatic receiving benralizumab - a case study. J Asthma. 2020;18:1-3. doi:10.1080/02770903.2020.1781165

58. Renner A, Marth K, Patocka K, Idzko M, Pohl W. COVID-19 in two severe asthmatics receiving benralizumab: busting the eosinophilia myth. ERJ Open Res. 2020;6(4):00457-2020. doi:10.1183/23120 541.00457-2020

59. Azim A, Pini L, Khakwani Z, et al. Severe acute respiratory syndrome coronavirus 2 infection in those on mepolizumab therapy. Ann Allergy Asthma Immunol. 2021;126(4):438-440. doi:10.1016/j.anai. 2021.01.006

60. Aksu K, Yesilkaya S, Topel M, et al. COVID-19 in a patient with severe asthma using mepolizumab. Allergy Asthma Proc. 2021;42(2): e55-e57. doi:10.2500/aap.2021.42.200125

61. Bhalla A, Mukherjee M, Radford K, et al. Dupilumab, severe asthma airway responses, and SARS-CoV-2 serology. Allergy. 2021;76 (3):957-958. doi:10.1111/all.14534

62. Global Initiative for Asthma (GINA). Global strategy for asthma management and prevention. Available from: https://ginasthma.org/. Accessed April 3, 2021.

63. Kerstjens HA, Moroni-Zentgraf P, Tashkin DP, et al. Tiotropium improves lung function, exacerbation rate, and asthma control, independent of baseline characteristics including age, degree of airway obstruction, and allergic status. Respir Med. 2016;117:198-206. doi:10.1016/j.rmed.2016.06.013
64. Virchow JC, Kuna P, Paggiaro P, et al. Single inhaler extrafine triple therapy in uncontrolled asthma (TRIMARAN and TRIGGER): two double-blind, parallel-group, randomised, controlled Phase 3 trials. Lancet. $\quad 2019 ; 394(10210): 1737-1749 . \quad$ doi:10.1016/S0140-6736(19) 32215-9

65. Liang Y, Chen M, Tan C, Tu C, Zheng X, Liu J. Successful sequential treatment for severe asthma coexisting COVID-19 via budesonide/glycopyrrolate/formoterol fumarate. Int J Gen Med. 2021;14:357-359. doi:10.2147/ijgm.s291695

66. Yamaya M, Nishimura H, Deng X, et al. Inhibitory effects of glycopyrronium, formoterol, and budesonide on coronavirus HCoV-229E replication and cytokine production by primary cultures of human nasal and tracheal epithelial cells. Respir Investig. 2020;58 (3):155-168. doi:10.1016/j.resinv.2019.12.005

67. NICE. COVID-19 rapid guideline: severe asthma. Available from: https://www.nice.org.uk/guidance/ng166. Accessed July 202021.

68. British Thoracic Society. Advice for healthcare professionals treating people with asthma (adults) in relation to COVID-19. Available from: https://www.brit-thoracic.org.uk. Accessed July 202021.

69. Bun S, Kishimoto K, Shin JH, et al. Impact of the COVID-19 pandemic on asthma exacerbations in children: a multi-center survey using an administrative database in Japan. Allergol Int. 2021;70(4):489-491. PMID: 34281768; PMCID: PMC8282991. doi:10.1016/j.alit.2021.06.001

70. World Allergy Organization (WAO). Available from: https://www. worldallergy.org/resources/covid-19. Accessed April 3, 2021.
ImmunoTargets and Therapy

\section{Publish your work in this journal}

ImmunoTargets and Therapy is an international, peer-reviewed open access journal focusing on the immunological basis of diseases, potential targets for immune based therapy and treatment protocols employed to improve patient management. Basic immunology and physiology of the immune system in health, and disease will be also covered. In addition, the journal will focus on the impact of management
Dovepress

programs and new therapeutic agents and protocols on patient perspectives such as quality of life, adherence and satisfaction. The manuscript management system is completely online and includes a very quick and fair peer-review system, which is all easy to use. Visit http://www.dovepress.com/testimonials.php to read real quotes from published authors. 